\title{
Dead Zone Compensation Method of H-Bridge Inverter Series Structure
}

\author{
Wei Li \\ Institute of Electrical Engineering and Information Engineering, Lanzhou University of Technology, Lanzhou 730050, China
}

\begin{abstract}
Based on the analysis of dead zone compensation principle in $\mathrm{H}$-bridge inverter series structure, an improved average voltage feedforward compensation method of $\mathrm{H}$-bridge inverter series structure is studied. Because the traditional average voltage feedforward compensation method has the phenomenon of false compensation in the current zero crossing region, the zero crossing region of the $\mathrm{H}$-bridge inverter series structure output current is precisely divided in the improved method. Outside the current zero-crossing region, adopting the average voltage feedforward method. During the current zero-crossing region, the dead zone will not affect the output voltage, so no compensation is required, which effectively avoid the traditional average voltage feedforward compensation method has the phenomenon of false compensation caused by misjudgment of current direction in zero crossing region. Finally, the improved dead zone compensation method of $\mathrm{H}$-bridge inverter series structure is simulated by MATLAB/SIMULINK software. The results verify the effectiveness of the method.
\end{abstract}

Keywords-dead zone; h-bridge ; inverter series; compensation method

\section{INTRODUCTION}

At present, the H-bridge inverter series structure has been applied in the electric car [1], photovoltaic grid-connected power generation system [2], series micro-grids [3] and wind/solar hybrid generation system [4] extensively. As the power switch has a certain turn-on and turn-off time, in order to prevent the phenomenon of short circuit appear in the bridge arm, dead time is needed to add to the power device switching process. The dead time, power device turn-on and turn-off time, the tube voltage drop lead to inverter output fundamental voltage loss and amplitudes of low harmonics increase, so resulting in the output voltage waveform and current waveform of the H-bridge inverter series structure distortion [5]. Therefore, it is necessary to compensate the output voltage waveform of the H-bridge inverter series structure.

At present, domestic and foreign scholars have proposed a variety of dead-zone compensation methods for single-phase or three-phase bridge inverters. Pulse width direct adjustment method [6], this method control the upper or lower switch tube that on the same arm closing early or opening delay in a little time by detecting the direction of the current to ensure that the upper and lower switch will not opening at the same time during the switching process, meanwhile the integrity of the output voltage waveform can also be ensured. Invalid switch elimination method [7], this method only allow one of the power switches that on the same bridge arm to act by judging the direction of the current, thus avoiding the phenomenon of " pass through" happened in the same arm, so there is not necessary to set the dead time. The Average voltage feedforward compensation method [8], this method calculate the average value of the difference between the actual output voltage waveform that considered the dead zone and the ideal output voltage waveform (the difference is dead zone pulse voltage), then compensate it to the modulation wave through the feedforward way. In this method, the compensation voltage is positive or negative depends on the direction of the inverter output current.

When the dead zone in H-bridge inverter series structure is compensated by the pulse width direct adjustment method or the invalid switch elimination method, the pulse width direct adjustment method need to adjustment every switch tube's driving signal of each inverter unit by detecting the direction of the current and the invalid switch elimination method also require masking every switch tube's invalid driving signal. While there are a number of switches in the $\mathrm{H}$-bridge inverter series structure that make the entire control system becomes very complex, so the compensation method will take up a lot of computing resources of the controller. Since all the inverter units of H-bridge inverter series structure share a modulation wave, applying the average voltage feedforward compensation method to H-bridge inverter series structure to complete the dead zone compensation for all inverter units only needs to compensate the modulation wave. So the average voltage feedforward compensation method is more suitable for the dead zone compensation of H-bridge inverter series structure. However, because in the current zero-crossing region of the $\mathrm{H}$-bridge inverter series structure, the current jumps between the positive and negative very frequently, to use the traditional average voltage feedforward compensation method is prone to result in false compensation by misjudgment of the current direction. Based on this problem, this paper studied an improved average voltage feedforward compensation method for the dead zone compensation problem of H-bridge inverter series structure that under carrier phase shift-SPWM modulation strategy. This improved method divides the zero-crossing region of the $\mathrm{H}$-bridge inverter series structure output current precisely. Outside the current zero-crossing region, compensate the average voltage to the modulation wave. In the zero-crossing region of the current, because the dead zone has no effect on the output voltage [9], compensation is not required anymore. The improved average voltage feedforward compensation method not only avoids the false compensation phenomenon caused by the misjudgment of the current direction in the current zero-crossing region, but also makes the dead zone compensation module in H-bridge inverter series structure more simplified, so the compensation module occupying the memory of the controller will be 
reduced. Above all, the improved average voltage feedforward compensation method has a great research significance.

II. The Calculation of DeAd Zone Compensation TIME IN H - BRIDGE INVERTER SERIES STRUCTURE

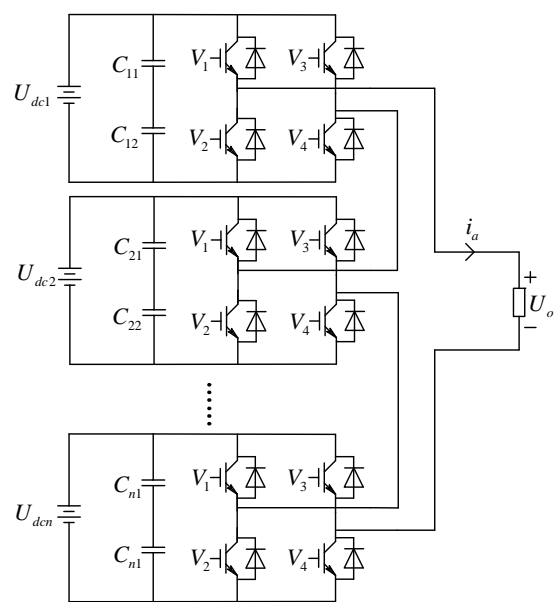

FIGURE I. MODEL OF H-BRIDGE INVERTER SERIES STRUCTURE

The circuit model of $\mathrm{H}$-bridge inverter series structure shown in figure I. It is assumed that each inverter unit has the same power devices. Taking the left arm switches of the $i$ th inverter unit as an example to analysis, and the analysis methods of remaining inverter units are the same.
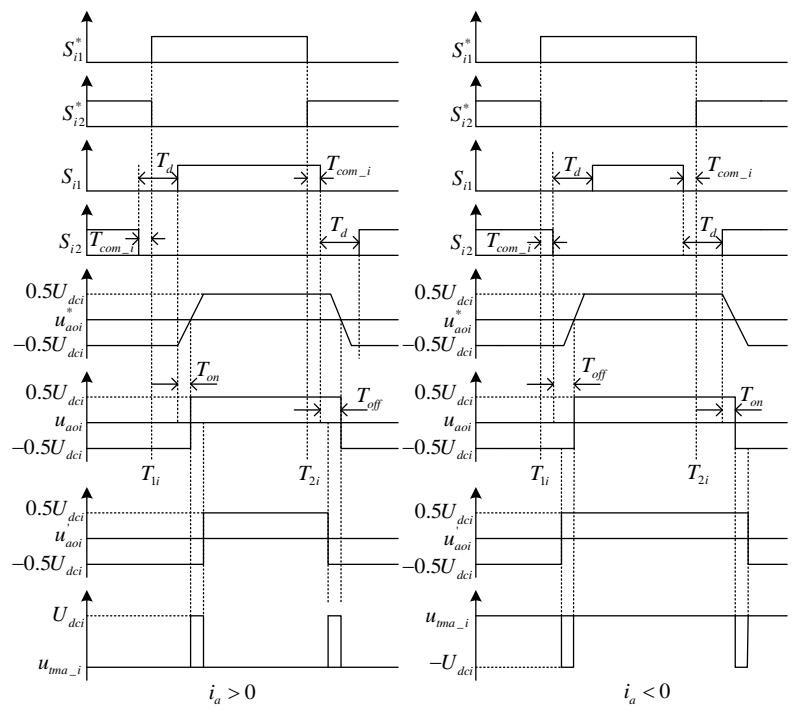

FIGURE II. PRINCIPLE OF DEAD ZONE COMPENSATION

Figure II is the analysis of dead zone compensation principle. Signals $S_{i 1}^{*}, S_{i 2}^{*}$ are respectively the ideal driving signals of the $i$ th inverter unit's switches $V_{1}, V_{2}$. Signals $S_{i 1}, S_{i 2}$ are respectively the actual driving signals of the $i$ th inverter unit's switches $V_{1}, V_{2} . u_{a o i}^{*}$ is the actual output voltage waveform of the $i$ th inverter unit left arm. $u_{\text {aoi }}$ is the equivalent output voltage waveform of the $i$ th inverter unit left arm. $u_{a o i}^{\prime}$ is the equivalent output voltage waveform of the $i$ th inverter unit left arm without the compensation. $u_{t m a_{-} i}$ ( $u_{t m a \_}=u_{a o i}-u_{a o i}^{\prime}$ ) is the compensation pulse voltage of the $i$ th inverter unit left arm. $T_{d}$ is the dead time. $T_{\text {com_i }}$ is the compensation pulse voltage width of the $i$ th inverter unit. $T_{\text {on }}$, $T_{\text {off }}$ are respectively the power switch turn-on time and turn-off time. $T_{1 i}, T_{2 i}$ are respectively the ideal opening time and ideal closing time of the $i$ th inverter unit's switch $V_{1}$; $U_{d c i}$ is the DC side voltage of the $i$ th inverter unit. $i_{a}$ is the output current of the H-bridge inverter series structure.

Assuming that the voltage drops across every power tube of the inverter units are the same, the voltage drops of the diodes are the same and the midpoint's voltages are balanced. According to the analysis method as in [10], it can be obtained that the actual output voltage of the $i$ th inverter unit in a switch period $T_{s}$ is:

$$
\begin{gathered}
u_{a b_{-} i}=\left(\frac{T_{a i}^{*}-T_{b i}^{*}}{T_{s}}\right) U_{d c i}+\left(\frac{2}{T_{s}} U_{d c i} T_{m i}-V_{d}-V_{c e}\right) \operatorname{sign}\left(i_{a}\right) \\
\operatorname{sign}\left(i_{a}\right)=\left\{\begin{array}{cc}
1 & i_{a}>0 \\
-1 & i_{a}<0
\end{array}\right.
\end{gathered}
$$

where $u_{a b_{-} i}$ is the output voltage of the $i$ th inverter unit. $T_{a i}^{*}$ is the ideal conduction time of the $i$ th inverter unit's power switch $V_{1}\left(T_{a i}^{*}=T_{2 i}-T_{1 i}\right) . T_{m i}$ is the time difference between the actual conduction and ideal conduction of the $i$ th inverter unit's power switch $V_{1}$ or $V_{3}\left(T_{m i}=T_{\text {off }}-T_{\text {on }}-T_{d}+2 T_{\text {com_ } i}\right)$. $T_{b i}^{*}$ is the ideal conduction time of the $i$ th inverter unit's power switch $V_{3} . V_{c e}$ is the voltage drop of the power switch. $V_{d}$ is the voltage drop of the freewheeling diode.

In equation (1), the first term is the ideal output voltage of the $i$ th inverter unit. In order to make the actual voltage equal to the ideal voltage, it is easy to know that the second term must be zero. So that there is:

$$
\left(\frac{2}{T_{s}} U_{d c i} T_{m i}-V_{d}-V_{c e}\right) \operatorname{sign}\left(i_{a}\right)=0
$$

The condition of equation (2) can be established is:

$$
\frac{2}{T_{s}} U_{d c i} T_{m i}-V_{d}-V_{c e}=0
$$

Substituting $T_{m i}=T_{\text {off }}-T_{\text {on }}-T_{d}+2 T_{\text {com_ }_{-} i}$ into equation (3), then transformed it can obtain:

$$
T_{\text {com_i } i}=\frac{T_{d}+T_{o n}-T_{o f f}}{2}+\frac{\left(V_{d}+V_{c e}\right) T_{s}}{4 U_{d c i}}
$$




\section{Dead Zone Compensation Method of H-BRidge INVERTER SERIES STRUCTURE}

The dead-zone compensation pulse voltage $u_{t m a \_}$in figure II can be equivalently replaced by a square wave, as shown in figure III.

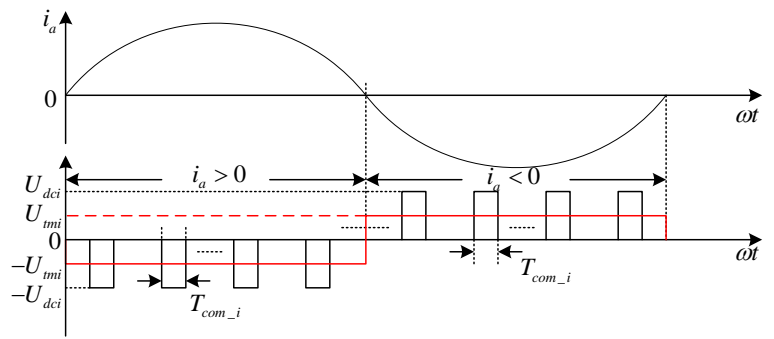

FIGURE III. WAVEFORM OF AVERAGE COMPENSATION VOLTAGE

The amplitude of square wave that has the same function with the dead zone compensation pulse voltage of the $i$ th inverter unit is:

$$
U_{t m i}=\frac{2 T_{c o m_{-} i}}{T_{s}} \times U_{d c i}
$$

When the inverter DC side voltages are equal:

$$
U_{d c 1}=U_{d c 2}=\cdots=U_{d c i}=U_{d c}(i=1,2, \cdots, n)
$$

From equation (4) can obtain:

$$
T_{\text {com_ } 1}=T_{\text {com_ } 2}=\cdots=T_{\text {com_ }_{-} i}=T_{\text {com }}(i=1,2, \cdots, n)
$$

The average compensation voltage amplitude of each inverter unit is:

$$
\begin{aligned}
U_{t m}= & U_{t m 1}=U_{t m 2}=\cdots=U_{t m i}(i=1,2, \cdots, n) \\
U_{t m} & =\frac{2 T_{c o m}}{T_{s}} \times U_{d c} \\
& =\frac{2 U_{d c}}{T_{s}} \times\left[\frac{T_{d}+T_{o n}-T_{\text {off }}}{2}+\frac{\left(V_{d}+V_{c e}\right) T_{s}}{4 U_{d c}}\right] \\
& =\frac{U_{d c}\left(T_{d}+T_{\text {on }}-T_{\text {off }}\right)}{T_{s}}+\frac{\left(V_{d}+V_{c e}\right)}{2}
\end{aligned}
$$

And then the compensation voltage that compensated on the modulation wave is:

$$
\begin{aligned}
& u_{t m}^{*}=\frac{U_{t m} \operatorname{sign}\left(i_{a}\right)}{U_{d c} / 2} \\
& =\left[\frac{2\left(T_{d}+T_{o n}-T_{o f f}\right)}{T_{s}}+\frac{\left(V_{d}+V_{c e}\right)}{U_{d c}}\right] \operatorname{sign}\left(i_{a}\right)
\end{aligned}
$$

In which, $u_{t m}^{*}$ is the compensation voltage.

When the carrier ratio $F$ is big, assuming that all the switching periods $T_{s}$ are equal, then:

$$
T_{s}=\frac{1}{f_{c}}
$$

In which, $f_{c}$ is the carrier frequency. Substituting equation (8) into equation (7) can obtain:

$$
u_{t m}^{*}=\left[2 f_{c}\left(T_{d}+T_{o n}-T_{o f f}\right)+\frac{\left(V_{d}+V_{c e}\right)}{U_{d c}}\right] \operatorname{sign}\left(i_{a}\right)
$$

It can be seen from Equation (9) that the compensation voltage is positive or negative depends on the judgment of the load current direction, so the accurate judgment of the current direction directly affects the accuracy of the compensation. In the current zero-crossing region, the dead zone does not affect the output voltage of the inverter, so no compensation is required. Only the part of the current zero-crossing region is needed to compensate to avoid the phenomenon of false compensation caused by misjudgment the current direction in the zero-crossing region. Therefore, the precise division of current zero-crossing region of the $\mathrm{H}$-bridge inverter series structure is particularly important. When the inverter unit switches $V_{1}, V_{4}$ turn on and switches $V_{2}, V_{3}$ turn off, the current of load rises. When switches $V_{1}, V_{4}$ turn off and switches $V_{2}, V_{3}$ turn on, the current of load drops. Therefore, it is necessary to find the switch positive or negative maximum conduction time of the $\mathrm{H}$-bridge inverter series structure in the current zero-crossing region.

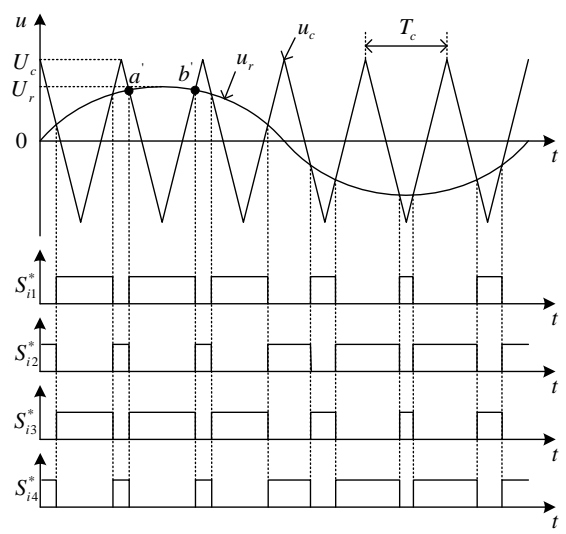

FIGURE IV. BIPOLAR SPWM MODULATION

In figure IV, $S_{i 1}^{*}, S_{i 2}^{*}, S_{i 3}^{*}, S_{i 4}^{*}$ are respectively the ideal driving signals of the $i$ th inverter unit's switches $V_{1}, V_{2}, V_{3}$, $V_{4} \cdot u_{r}$ is the modulation wave. $u_{c}$ is the carrier wave. $U_{r}$ is the amplitude of modulation wave. $U_{c}$ is the amplitude of carrier wave. $T_{c}$ is the carrier wave period. When the modulation wave $u_{r}$ is bigger than carrier wave $u_{c}$, the 
switches $V_{1}, V_{4}$ turn on. When the modulation wave $u_{r}$ is less than carrier wave $u_{c}$, the switches $V_{2}, V_{3}$ turn on. Because of symmetry, the maximum turn on time of the switches $V_{1}, V_{4}$ is also the maximum turn on time of the switches $V_{2}, V_{3}$. As shown in figure IV, the triangular wave that is bipolar carrier can be expressed as follows:

$$
u_{c}=\left\{\begin{array}{cc}
-\frac{4 U_{c}}{T_{c}}\left(t-k T_{c}\right)+U_{c} & k T_{c} \leq t \leq k T_{c}+\frac{T_{c}}{2} \\
\frac{4 U_{c}}{T_{c}}\left(t-k T_{c}-\frac{T_{c}}{2}\right)-U_{c} & k T_{c}+\frac{T_{c}}{2} \leq t \leq k T_{c}+T_{c}
\end{array}\right.
$$

In which, $k=0,1,2,3, \cdots$.

The equation of sine modulation wave is:

$$
u_{r}=U_{r} \sin \omega_{r} t
$$

So that making the modulation ratio $M=U_{r} / U_{c} \leq 1$ and making the modulation amplitude $U_{c}$ remain constant. At the sampling point $a^{\prime}$, there is $u_{r}=u_{c}$, then:

$$
U_{r} \sin \omega_{r} t=-\frac{4 U_{c}}{T_{c}}\left(t_{1}-k T_{c}\right)+U_{c}
$$

From the equation (12) can obtain:

$$
t_{1}=\left(k+\frac{1}{4}-\frac{1}{4} M \sin \omega_{r} t\right) T_{c}
$$

Similarly, at the sampling point $b^{\prime}$ there is:

$$
U_{r} \sin \omega_{r} t=\frac{4 U_{c}}{T_{c}}\left(t_{2}-k T_{c}-\frac{T_{c}}{2}\right)-U_{c}
$$

Solved equation (14) can obtain:

$$
t_{2}=\left(k+\frac{3}{4}+\frac{1}{4} M \sin \omega_{r} t\right) T_{c}
$$

So the conduction time of switches is:

$$
\begin{aligned}
& \Delta t^{*}=t_{2}-t_{1} \\
& =\frac{1}{2}\left(1+M \sin \omega_{r} t\right) T_{c}
\end{aligned}
$$

Because the current waveform lag voltage waveform $\varphi$ in phase, $\varphi$ is the power factor angle. Therefore, in the current zero-crossing, switches conduction time can be expressed as follows:

$$
\begin{aligned}
& \Delta t^{*}=t_{2}-t_{1} \\
& =\frac{1}{2}(1+M \sin \varphi) T_{c}
\end{aligned}
$$

In the carrier phase shift-SPWM modulation mode, the maximum time that only one inverter unit's switches act, the remaining inverter unit's switches remain static is:

$$
\begin{aligned}
\Delta t & =\frac{\Delta t^{*}}{N} \\
& =\frac{1}{2 N}(1+M \sin \varphi) T_{c}
\end{aligned}
$$

When the current crosses zero, the voltage amplitude of the load equivalent resistor is:

$$
U_{R}=N M U_{d c} \sin \varphi
$$

Therefore, at the current zero-crossing point, the voltage amplitude of the load equivalent inductance $L$ is:

$$
\begin{aligned}
U_{L} & =U_{d c}-U_{R} \\
& =U_{d c}-N M U_{d c} \sin \varphi \\
& =U_{d c}(1-N M \sin \varphi)
\end{aligned}
$$

And because of $U_{L}=L \frac{\Delta i}{\Delta t}$, there is:

$$
\Delta i=\frac{U_{L}}{L} \Delta t
$$

Substituting equation (18) and equation (20) into equation (21), the variation of current can be obtained as follows:

$$
\Delta i=\frac{U_{d c}(1-N M \sin \varphi)}{2 N L}(1+M \sin \varphi) T_{c}
$$

In the current zero-crossing region, the positive or negative maximum value of the current is less than the current variation $\Delta i$. When the load current is $i_{a} \in(-\Delta i, \Delta i)$, there is no dead zone compensation. When the load current is $i_{a} \leq-\Delta i$ or $i_{a} \geq \Delta i$, the compensation voltage that calculated from equation (9) will be compensated to the ideal modulation wave. The compensation voltage expression in different current region is:

$$
u_{t m}^{*}=\left\{\begin{array}{cc}
{\left[2 f_{c}\left(T_{d}+T_{\text {on }}-T_{\text {off }}\right)+\frac{\left(V_{d}+V_{c e}\right)}{U_{d c}}\right]} & i_{a} \geq \Delta i \\
0 & \left|i_{a}\right|<\Delta i \\
-\left[2 f_{c}\left(T_{d}+T_{\text {on }}-T_{\text {off }}\right)+\frac{\left(V_{d}+V_{c e}\right)}{U_{d c}}\right] & i_{a} \leq-\Delta i
\end{array}\right.
$$


The diagram of the compensation method is shown in the following figure:

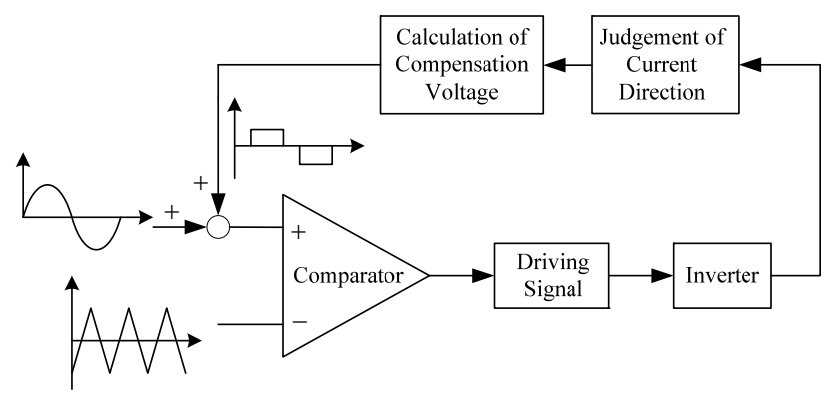

FIGURE V. DIAGRAM OF THE COMPENSATION METHOD

\section{SIMULATION ANALYSIS}

In this paper, take the five-cell H-bridge inverter series structure as an example to simulate. The DC side voltage is $U_{d c}=300 \mathrm{~V}$. The frequency of modulation wave is $f_{c}=2000$ Hz. Load resistance is $R=10 \Omega$. Load inductance is $L=3$ $\mathrm{mH}$. Dead time is $T_{d}=2 \times 10^{-5} \mathrm{~s}$. Modulation ratio is $M=0.8$. IGBT turn-on and turn-off time are respectively $t_{\text {on }}=1 \mu \mathrm{s}, t_{\text {off }}=1.2 \mu \mathrm{s}$. Voltage drop of the IGBT in conduction is $V_{c e}=2 \mathrm{~V}$. Voltage drop of the freewheeling diode in conduction is $V_{d}=2.5 \mathrm{~V}$. Substituting all above parameters into the equation (22) and equation (23) can obtain:

$$
\begin{gathered}
\Delta i \approx 3.35 \mathrm{~A} \\
u_{t m}^{*}=\left\{\begin{array}{cc}
0.0942 & i_{a} \geq 3.35 \\
0 & \left|i_{a}\right|<3.35 \\
-0.0942 & i_{a} \leq-3.35
\end{array}\right.
\end{gathered}
$$

The simulation results are shown as follows:

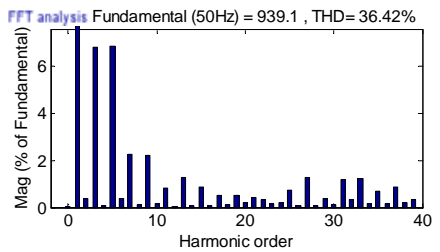

(A) BEFORE COMPENSATION

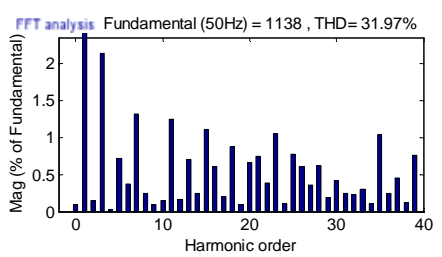

(B) AFTER COMPENSATION

FIGURE VI. SPECTRUM OF THE OUTPUT VOLTAGE

Figure VI(a) is the spectrum of the five-cell H-bridge inverter series structure output voltage that considered turn-on time of the power switch, turn off time of the power switch, voltage drop of the power switch, voltage drop of the freewheeling diode and dead time. Figure VI(b) is the spectrum of the output voltage after compensation. It can be seen from figure VI, after take the compensation method, the amplitude of fundamental voltage increased from $939.1 \mathrm{~V}$ to $1138 \mathrm{~V}$. At the same time, the low harmonics amplitudes decrease and the waveform distortion rate decreases from $36.42 \%$ to $31.97 \%$.

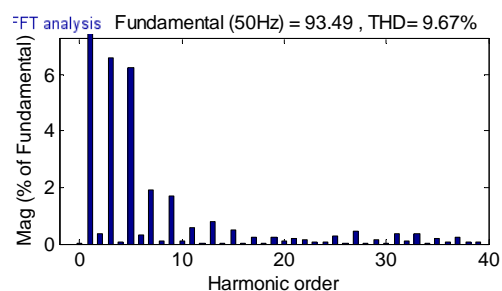

(A) BEFORE COMPENSATION

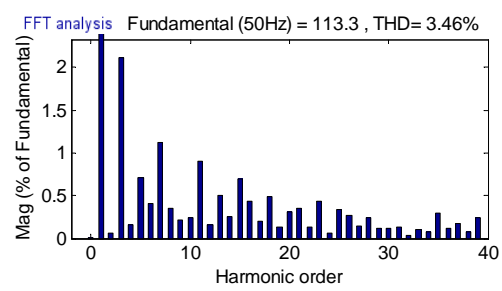

(B) AFTER COMPENSATION

FIGURE VII. SPECTRUM OF THE OUTPUT CURRENT

Figure VII (a) shows the spectrum of the five-cell H-bridge inverter series structure output current before compensation. Figure VII(b) is the spectrum of the output current after compensation. It can be seen from figure VII, after adopting the compensation method, the amplitude of the fundamental current increased from 93.49A to 113.3A. At the same time, the low harmonics amplitudes decrease and the current waveform distortion rate decreases from $9.67 \%$ to $3.46 \%$.

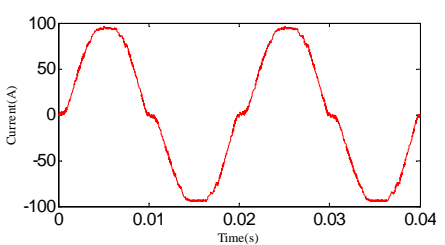

(A) BEFORE COMPENSATION

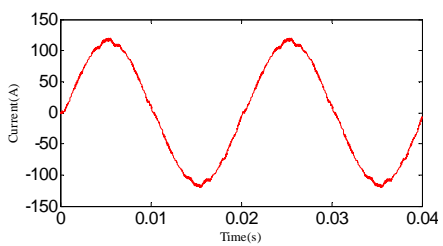

(B) AFTER COMPENSATION

FIGURE VIII. OUTPUT CURRENT WAVEFORM

Figure VIII(a) shows the output current waveform of the five-cell H-bridge inverter series structure before compensation. Figure VIII(b) is the output current waveform after compensation. It can be seen from figure VIII, after adopting the compensation method, the output current waveform of the five-cell $\mathrm{H}$-bridge inverter series structure became more better. 


\section{CONCLUSION}

In this paper, an improved average voltage feedforward compensation method of H-bridge inverter series structure that using the CPS-SPWM modulation strategy was studied. As the $\mathrm{H}$-bridge inverter series structure in the CPS-SPWM modulation mode, there are power switches of the inverter unit act every $T_{c} / N$. So in the current zero-crossing region, $\mathrm{H}$-bridge inverter series structure output current jumps between the positive and negative very frequently. In this method, the output current zero-crossing region of H-bridge inverter series structure is precisely divided. In the current zero-crossing region, dead zone compensation is not needed. Outside the current zero-crossing region, The compensation voltage amplitude is calculated to complete the dead zone compensation. The improved method solved the phenomenon of false compensation that caused by the traditional average voltage feedforward compensation method due to the misjudgment of the current direction in the current zero-crossing region. The simulation results show that the improved method can effectively decrease the influence of the dead zone on output voltage and current of the H-bridge inverter series structure.

\section{REFERENCES}

[1] S. H. Hosseini, M. Ahmadi, S. G. Zadeh, "reducing the output harmonic of cascade H-bridge multilevel inverter for electric vehicle applications," 1rd ed, Electrical Power System, 2011, pp. 752-755.

[2] B. Xiao, L. Hang, J. Mei, C. Riley, L. M. Tolbert, B. Ozpineci, "Modular Cascaded H-Bridge Multilevel PV Inverter With Distributed MPPT for Grid-Connected Applications,” 2rd ed, vol. 51, IEEE Transactions on Industry Applications, 2015, pp. 1722-1731.

[3] X. Wang, W. Yang, "Dynamic modeling of inverter system in isolated series micro-grid,” 8rd ed, vol. 40, High Voltage Engineering, 2014, pp. 2456-2463.

[4] X. Wang, Q. Guo, W. Yang, "Scenery complementary power generation system based on inverter series power balance control," 11rd ed, vol. 38, Chinese Journal of Power Sources, 2014, PP. 2152-2191.

[5] A. R. Munoz, T. A. Lipo, "On-line dead-time compensation technique for open-loop PWM-VSI drives,” 4rd ed, vol. 14, IEEE Transactions on Power Electronics, 1999, pp. 683-689.

[6] C. Attaianese, V. Nardi, T. Giuseppe, "A novel SVM strategy for VSI dead-time-effect reduction,” 2rd ed, vol. 51, IEEE Transactions on Industry Applications, 2015, pp. 1722-1731.

[7] L. H. Chen, F. Z. Peng, "Dead-time elimination for voltage source inverters," 2rd ed, vol. 23, IEEE Transactions on Power Electronics, 2008, pp. 574-580.

[8] T. Sukegawa, K. Kamiyama, K. Mizuno, T. Matsui, T. Okuyama, "Fully digital vector-controlled PWM VSI-fed ac drives with an inverter dead-time compensation strategy,” 3rd ed, vol. 27, IEEE Transactions on Industry Applications, 1991, pp. 552-559.

[9] B. Yang, J. Wu, W. Li, X. He, “Online adaptive dead-time elimination method for PWM voltage source inverters," 11rd ed, vol. 26, Transactions of China Electrotechnical Society, 2011, pp. 45-52.

[10] L. Yang, Z. Chen, W. Chen, Z. Li, “Output voltage model of VSI-inverter and a novel dead-Time compensation method,” 1rd ed, vol. 27, Transactions of China Electrotechnical Society, 2012, pp. 182-187. 